\title{
Comparação das Medidas da Área Valvar Mitral Obtidas por Parâmetros Hemodinâmicos Invasivos e Ecocardiografia Tridimensional em Tempo Real Pré e Pós-Valvoplastia Mitral Percutânea
}

\author{
Luis Augusto Palma Dallan'1, Marcelo Luiz Campos Vieira' ${ }^{1}$, Murilo Capreti da Silva', \\ Camila Rebouças Wagner ${ }^{1}$, Luiz Junya Kajita ${ }^{1}$, Rogerio Miranda ${ }^{1}$, Flávio Tarasoutchi ${ }^{1}$, \\ Luiz Francisco Cardoso', Max Grinberg ${ }^{1}$, Wilson Mathias Jr. ${ }^{1}$, \\ Carlos Alberto Pastore', Eulogio Emilio Martinez ${ }^{1}$
}

\section{RESUMO}

Introdução: A técnica de ecocardiografia tridimensional em tempo real (eco-3D) para avaliação da área valvar mitral (AVM) é uma técnica inovadora, que deve ser validada na comparação com técnicas de cálculos de AVM já consagrados. O cálculo da AVM pelo método de Gorlin já foi bem estabelecido e correlaciona-se de forma adequada com a verdadeira AVM. Nosso objetivo foi comparar as medidas de AVM obtidas pelos métodos hemodinâmico e ecocardiográfico, a partir de parâmetros hemodinâmicos invasivos (fórmula de Gorlin) e de eco-3D após valvoplastia mitral percutânea por cateter-balão (VCB). Método: Estudo prospectivo, realizado entre fevereiro de 2009 e fevereiro de 2010, em que foram selecionados 24 indivíduos (dos quais 22 mulheres, na faixa etária de $39 \pm 12$ anos) portadores de estenose mitral sintomática submetidos a VCB. Foi feita análise ecocardiográfica transtorácica bidimensional e tridimensional antes e após a VCB, em que foram aferidos a AVM (eco-3D/medida invasiva) e o gradiente mitral (máximo/médio). Resultados: Foram excluídos 2 pacientes (um por trombo em átrio esquerdo e outro por AVM $>1,5 \mathrm{~cm}^{2}$ ). A média da AVM obtida pelo cálculo hemodinâmico foi de $1,73 \mathrm{~cm}^{2}$, enquanto a média obtida pela eco-3D foi de $1,72 \mathrm{~cm}^{2}$. As correlações entre as AVMs obtidas pelo método de Gorlin e pela eco-3D pré-valvoplastia (r: 0,7638; $\mathrm{P}<0,001)$ e pós-valvoplastia ( $\mathrm{r}: 0,6659$; $P<0,0019)$ foram significativas. Conclusão: A eco-3D é

\section{ABSTRACT}

Comparison of Mitral Valve Area Measurements by Invasive Hemodynamic Parameters and Real-Time Three-Dimensional Echocardiography Before and After Percutaneous Mitral Valvuloplasty

Background: Real time three-dimensional (3D) echocardiography (Echo) is an innovative technique to assess mitral valve area (MVA) that must be validated in comparison with established MVA calculation techniques. The calculation of valve area by Gorlin's method has been well established and correlates adequately with the true MVA. This study was aimed at comparing MVA obtained by hemodynamic and echocardiographic methods, based on invasive hemodynamic (Gorlin's formula) and 3D Echo parameters after percutaneous balloon mitral valvuloplasty (PBMV). Method: Prospective study carried out from February 2009 to February 2010, in 24 individuals (22 women, $39 \pm$ 12 years) with symptomatic mitral stenosis undergoing PBMV. $2 \mathrm{D}$ and $3 \mathrm{D}$ Echo were compared before and after the PBMV, with MVA (3D Echo/invasive measurement) and mitral gradient (max/mean) measurements. Results: Two patients were excluded (one because of a left atrial thrombus and another due to MVA $>1.5 \mathrm{~cm}^{2}$ ). Mean MVA obtained by the hemodynamic method was $1.73 \mathrm{~cm}^{2}$, whereas the mean value obtained by $3 \mathrm{D}$ Echo was $1.72 \mathrm{~cm}^{2}$. There was a significant correlation between MVA obtained by

1 Instituto do Coração do Hospital das Clínicas da Faculdade de Medicina da Universidade de São Paulo (InCor/HCFMUSP) - São Paulo, SP, Brasil.

Correspondência: Luis Augusto Palma Dallan. Av. Dr. Enéas Carvalho de Aguiar, 44 - Cerqueira César - São Paulo, SP, Brasil CEP 05403-000

E-mail: gutodallan@hotmail.com

Recebido em: 15/8/2010 • Aceito em: 28/9/2010 
um exame adequado para avaliação e cálculo da AVM pré e pós-VCB, com valor fidedigno quando comparado ao consagrado cálculo da AVM obtido pela fórmula de Gorlin.

DESCRITORES: Estenose da valva mitral. Dilatação por balão. Ecocardiografia. Ecocardiografia tridimensional.

A técnica de ecocardiografia tridimensional em tempo real para avaliação da área valvar é uma técnica inovadora, que deve ser validada na comparação com técnicas de cálculos de área valvar já consagrados. ${ }^{1-8} \mathrm{O}$ cálculo da área valvar pelo método de Gorlin já foi bem estabelecido e correlaciona-se de forma adequada com a verdadeira área valvar mitral. ${ }^{9-13}$

A ecocardiografia tem se caracterizado por ser método diagnóstico de escolha para o estudo morfofuncional das estruturas cardíacas em razão de sua grande correspondência anatômica, facilidade de execução, baixo custo, ausência de radiação ionizante e boa reprodutibilidade. No entanto, a ecocardiografia bidimensional, técnica atualmente mais utilizada na análise cardíaca estrutural, apresenta limitações para a análise da anatomia cardíaca. Esta análise limitada é decorrente das inferências geométricas assumidas para o cálculo dos diâmetros e volumes cardíacos a partir de limitado número de planos de observação. ${ }^{1-8}$ Situações de maior divergência anatômica podem ocorrer na eventualidade de dilatação das câmaras cardíacas. Dessa forma, foi desenvolvido a ecocardiografia tridimensional, que permite a visibilização estrutural considerando-se múltiplos planos simultâneos de observação, proporcionando maior proximidade com a anatomia cardíaca.

A validação da análise da área valvar mitral com o emprego da ecocardiografia tridimensional tem sido demonstrada em estudos prévios ${ }^{1-8}$, mas poucos estudos na literatura validaram a área valvar mitral pré e pósvalvoplastia mitral percutânea por cateter-balão por esse método, particularmente utilizando cálculos hemodinâmicos invasivos como referência.

O objetivo deste estudo foi comparar as medidas de área valvar mitral entre os métodos hemodinâmico e ecocardiográfico, por meio da fórmula de Gorlin e dos parâmetros obtidos na ecocardiografia tridimensional, após procedimento de valvoplastia mitral percutânea por cateter-balão, com o intuito de realizar a validação do método de ecocardiografia tridimensional a partir de cálculos hemodinâmicos invasivos consagrados.

\section{MÉTODO}

Estudo realizado no período de fevereiro de 2009 a fevereiro de 2010, no Instituto do Coração do Hos-
Gorlin's formula and 3D Echo pre-valvuloplasty ( $\mathrm{r}: 0.7638$; $\mathrm{P}<0.001)$ and post-valvuloplasty ( $\mathrm{r}: 0.6659 ; \mathrm{P}<0.0019)$. Conclusion: 3D Echo is an adequate method for the assessment and calculation of MVA before and after PBMV procedures providing accurate values when compared to the established MVA calculation obtained by Gorlin's formula.

KEY-WORDS: Mitral valve stenosis. Balloon dilatation. Echocardiography. Three-dimensional echocardiography.

pital das Clínicas da Faculdade de Medicina da Universidade de São Paulo (InCor/HCFMUSP), após a aprovação do Comitê de Ética em Pesquisa da instituição. Foram selecionados, de forma consecutiva e prospectiva, 24 indivíduos (22 mulheres, idade $39 \pm 12$ anos) portadores de estenose mitral sintomática submetidos a valvoplastia mitral percutânea por cateter-balão.

\section{Ecocardiografia tridimensional}

A aquisição de imagens tridimensionais foi realizada na sequência do exame ecocardiográfico bidimensional. As imagens foram obtidas com o equipamento IE 33 (Philips, Andover, Estados Unidos), com o emprego de transdutor matricial, estando o paciente em apneia expiratória, tendo sido a imagem acoplada ao registro eletrocardiográfico. As imagens foram armazenadas no disco rígido do aparelho ecocardiográfico e analisadas off-line em software específico do próprio equipamento (Q Lab, versão 6.0, Philips, Andover, Estados Unidos). As análises foram realizadas por dois observadores independentes.

Foi feita análise ecocardiográfica transtorácica bidimensional e tridimensional antes e após valvoplastia mitral percutânea por cateter-balão, em que foram aferidos a área valvar mitral [planimetria bidimensional, pressure half time ( $\mathrm{PHT})$, ecocardiografia tridimensional, medida invasiva] e o gradiente mitral (máximo/médio).

\section{Parâmetros hemodinâmicos invasivos}

A área valvar mitral $\left(\mathrm{cm}^{2}\right)$ foi obtida pela fórmula descrita por Gorlin e Gorlin:

$$
\mathrm{AVM}=\mathrm{DC} /(\mathrm{PED} \times \mathrm{FC}) \div(37,7 \times \text { [raiz quadrada } \Delta \mathrm{P}])
$$

em que AVM = área valvar mitral, DC = débito cardía$\mathrm{co}\left(\mathrm{cm}^{3}\right.$ por minuto $), \mathrm{FC}=$ frequência cardíaca, $\mathrm{PED}=$ período de enchimento diastólico (segundos por batida), e $\Delta \mathrm{P}=$ gradiente médio de pressão diastólica entre átrio esquerdo e ventrículo esquerdo $(\mathrm{mmHg})$.

\section{Análise estatística}

Foram realizadas análises descritivas de correlação e de comparação entre métodos. A análise descritiva das variáveis contínuas foi feita pela observação dos valores mínimos e máximos e do cálculo de médias e desvios padrão. A análise de correlação foi feita pelo 
método de correlação de Pearson (r), intervalo de confiança de $95 \%$. A comparação entre métodos foi realizada com o emprego do teste de Bland \& Altman. Foi também realizado teste de regressão linear entre os parâmetros aferidos com as ecocardiografias bidimensional e tridimensional. As medidas foram realizadas por dois observadores independentes. Valores de $\mathrm{P}<0,05$ foram considerados significantes. Os dados foram processados com o sistema de análise estatística MedCalc (Mariakerke, Bélgica).

\section{RESULTADOS}

A análise descritiva das características ecocardiográficas e hemodinâmicas da população estudada é demonstrada na Tabela 1.

Foram excluídos 2 pacientes (um por evidência de trombo em átrio esquerdo, observado durante a injeção de contraste no átrio esquerdo após a punção transeptal, e outro durante o procedimento hemodinâmico, por apresentar área valvar mitral $>1,5 \mathrm{~cm}^{2}$ ).

A média da área valvar mitral obtida pelo cálculo hemodinâmico foi de $1,73 \mathrm{~cm}^{2}$, enquanto a média obtida pela ecocardiografia tridimensional foi de $1,72 \mathrm{~cm}^{2}$.

A correlação entre a área valvar mitral obtida pela fórmula de Gorlin e a obtida pela ecocardiografia tridimensional, pré ( $r$ : 0,7638; $\mathrm{P}<0,001)$ e pós-valvoplastia (r: 0,6659; $\mathrm{P}<0,0019)$, foi significativa (Figuras 1 a 4).

\section{DISCUSSÃO}

A valvoplastia mitral percutânea por cateter-balão atualmente é o procedimento de escolha em pacientes com estenose mitral pura, sintomáticos, quando a valva mitral não é muito calcificada e a regurgitação mitral não é significativa, por ser um método seguro e custo-efetivo. Também é indicado para pacientes com alto risco cirúrgico. Os resultados da valvoplastia mitral percutânea por cateter-balão são equivalentes aos do tratamento cirúrgico por via aberta/comissurotomia, e ambos apresentam melhores resultados que a comissurotomia fechada. ${ }^{14}$

A determinação da área valvar mitral pode ser realizada com o emprego de diferentes técnicas nãoinvasivas de análise por imagem. Cada técnica apresenta particularidades específicas, e seu emprego tem vantagens e limitações. Atualmente a ecocardiografia bidimensional é o método mais amplamente utilizado para a análise anatômica e funcional cardíaca. Permite a avaliação anatômica das diversas estruturas cardíacas, possibilita a análise da diástole cardíaca com o emprego das diferentes modalidades de ecocardiografia Doppler, podendo ainda ser realizada à beira do leito em ambiente de terapia intensiva e de emergência. Apresenta, no entanto, limitações em razão de imagem ecocardiográfica subótima em pacientes obesos e em portadores de doença pulmonar obstrutiva crônica. Além disso, é relativamente dependente da interpretação do operador e limitado pelo emprego de pequeno número de planos anatômicos para a análise cardíaca.

O emprego da ecocardiografia tridimensional amplia a possibilidade de observação cardíaca ecocardiográfica a partir do exame estrutural, levando em consideração múltiplos planos espaciais de análise. A análise estrutural é realizada a partir de novos planos de identificação, sem que haja necessidade do emprego de extrapolações geométricas para a análise de formas anatômicas complexas (exemplo: ventrículo direito), e que nem sempre se limitam à formulação matemática específica. No entanto, a ecocardiografia tridimensional necessita de treinamento ecocardiográfico específico, requer estabilidade do ritmo cardíaco para sua melhor análise e também depende da qualidade de imagem inicial para seu melhor resultado.

Os resultados deste estudo, inédito no Brasil, permitem a validação do método de ecocardiografia tridimensional, a partir da medida invasiva (uma das mais exatas medidas disponíveis atualmente). A medida ecocardiográfica tridimensional possibilita a visão anatômica mais ampla da valva mitral, de seu aparato subvalvar, assim como do anel mitral. Dessa forma, possibilita a melhor identidade estrutural da complexa anatomia da valva mitral. ${ }^{1-8}$

A válvula cardíaca normal oferece pouca resistência ao fluxo sanguíneo. Conforme a estenose valvar se desenvolve, há cada vez mais resistência ao fluxo, causando um gradiente de pressão através da válvula. Em qualquer tamanho de orifício estenótico, quanto maior for o fluxo através do orifício maior será o gradiente de pressão.

No laboratório de cateterismo cardíaco, a gravidade da estenose mitral, que se reflete no gradiente transvalvar mitral médio, é medida durante a diástole, por meio da comparação simultânea de pressão do ventrículo esquerdo, obtido com um cateter do ventrículo esquerdo posicionado retrogradamente na aorta, e de pressão atrial esquerda, medida diretamente com um cateter transeptal ou indiretamente com um cateter na artéria pulmonar na posição firmada (pressão capilar pulmonar). ${ }^{9-11}$

O gradiente valvar médio é a diferença entre a pressão média do átrio esquerdo e a pressão média do ventrículo esquerdo durante a diástole. O período de enchimento diastólico e a frequência cardíaca são importantes variáveis na determinação do gradiente valvar mitral, como pode ser observado na fórmula de Gorlin. O gradiente é maior com uma frequência cardíaca mais alta e menor com uma frequência mais baixa, no último caso porque há mais tempo disponível para o enchimento do átrio esquerdo com um período prolongado de diástole. ${ }^{12}$

Entre os pacientes com fibrilação atrial, a gravidade da estenose mitral, avaliada por meio da pressão capilar pulmonar, pode ser diferente daquela obtida com a medida direta da pressão atrial esquerda. Pacientes que 
TABELA 1

Características ecocardiográficas e hemodinâmicas da população estudada

\begin{tabular}{|c|c|c|c|c|c|c|}
\hline & $\begin{array}{c}\text { Valvoplastia } \\
\text { mitral } \\
\text { percutânea }\end{array}$ & $\begin{array}{c}\text { Débito } \\
\text { cardíaco } \\
\text { (l/min) }\end{array}$ & $\begin{array}{c}\text { Frequência } \\
\text { cardíaca } \\
\text { (bpm) }\end{array}$ & $\begin{array}{c}\text { Gradiente } \\
\text { PD2VE-AE } \\
(\mathrm{mmHg})\end{array}$ & $\begin{array}{c}\text { Área valvar } \\
\text { (Gorlin) } \\
\left(\mathrm{cm}^{2}\right)\end{array}$ & $\begin{array}{c}\text { Área valvar } \\
\text { (eco 3D) } \\
\left(\mathrm{cm}^{2}\right)\end{array}$ \\
\hline \multirow[t]{2}{*}{1} & Pré & 3,2 & 68 & 10 & 0,67 & 1 \\
\hline & Pós & 2,9 & 78 & 2 & 1,36 & 1,55 \\
\hline \multirow[t]{2}{*}{2} & Pré & 2,9 & 75 & 8 & 0,68 & 0,9 \\
\hline & Pós & 3,4 & 71 & 2 & 1,6 & 1,8 \\
\hline \multirow[t]{2}{*}{3} & Pré & 6,4 & 83 & 12 & 1,23 & 1,5 \\
\hline & Pós & 6,4 & 88 & 4 & 2,14 & 2,2 \\
\hline \multirow[t]{2}{*}{4} & Pré & 3,4 & 78 & 8 & 0,8 & 1 \\
\hline & Pós & 4 & 78 & 4 & 1,33 & 1,5 \\
\hline \multirow[t]{2}{*}{5} & Pré & 4,2 & 93 & 7 & 1 & 1,1 \\
\hline & Pós & 4,1 & 83 & 3 & 1,54 & 1,9 \\
\hline \multirow[t]{2}{*}{6} & Pré & 3,7 & 76 & 13 & 1 & 1,1 \\
\hline & Pós & 4,5 & 74 & 7 & 1,7 & 1,7 \\
\hline \multirow[t]{2}{*}{7} & Pré & 5,9 & 83 & 15 & 1 & 1 \\
\hline & Pós & 7,7 & 83 & 5 & 2,3 & 1,7 \\
\hline \multirow[t]{2}{*}{8} & Pré & 3,1 & 75 & 17 & 0,5 & 1 \\
\hline & Pós & 4,1 & 70 & 4 & 1,35 & 1,2 \\
\hline \multirow[t]{2}{*}{9} & Pré & 4,1 & 80 & 8 & 0,94 & 1,1 \\
\hline & Pós & 4,2 & 80 & 4 & 1,3 & 1,3 \\
\hline \multirow[t]{2}{*}{10} & Pré & 5 & 100 & 7 & 1,25 & 1,2 \\
\hline & Pós & 5,3 & 100 & 2 & 2,5 & 1,8 \\
\hline \multirow[t]{2}{*}{11} & Pré & 5,6 & 77 & 8 & 1,97 & 1,4 \\
\hline & Pós & 5,6 & 73 & 3 & 3,2 & 1,2 \\
\hline \multirow[t]{2}{*}{12} & Pré & 5,1 & 88 & 11 & 1 & 1,3 \\
\hline & Pós & 3,6 & 88 & 2 & 1,7 & 2 \\
\hline \multirow[t]{2}{*}{13} & Pré & 5,6 & 93 & 25 & 0,7 & 0,9 \\
\hline & Pós & 5,4 & 83 & 3 & 2 & 1,7 \\
\hline \multirow[t]{2}{*}{14} & Pré & 4,5 & 75 & 13 & 0,83 & 1,2 \\
\hline & Pós & 5,6 & 68 & 4 & 1,88 & 2 \\
\hline \multirow[t]{2}{*}{15} & Pré & 4,9 & 83 & 15 & 0,84 & 1,3 \\
\hline & Pós & 5 & 83 & 5 & 1,5 & 1,8 \\
\hline \multirow[t]{2}{*}{16} & Pré & 4,3 & 78 & 20 & 0,65 & 0,8 \\
\hline & Pós & 4,1 & 65 & 5 & 1,22 & 1,6 \\
\hline \multirow[t]{2}{*}{17} & Pré & 2,6 & 75 & 15 & 0,45 & 0,8 \\
\hline & Pós & 4 & 83 & 6 & 1,1 & 1,4 \\
\hline \multirow[t]{2}{*}{18} & Pré & 4,3 & 70 & 12 & 0,83 & 0,95 \\
\hline & Pós & 4,1 & 75 & 2 & 1,93 & 1,6 \\
\hline \multirow[t]{2}{*}{19} & Pré & 2,8 & 125 & 18 & 0,44 & 1,2 \\
\hline & Pós & 3,1 & 75 & 6 & 0,84 & 1,45 \\
\hline \multirow[t]{2}{*}{20} & Pré & 5,6 & 75 & 17 & 0,91 & 1,08 \\
\hline & Pós & 5,1 & 78 & 3 & 1,96 & 1,8 \\
\hline \multirow[t]{2}{*}{21} & Pré & 3,3 & 75 & 16 & 0,8 & 0,6 \\
\hline & Pós & 4,7 & 100 & 4 & 2,35 & 1,8 \\
\hline \multirow[t]{2}{*}{22} & Pré & 4,6 & 80 & 20 & 1 & 0,9 \\
\hline & Pós & 5,1 & 78 & 7 & 2 & 1,9 \\
\hline
\end{tabular}

eco 3D = ecocardiografia tridimensional; PDVE-AE = pressão diastólica entre ventrículo esquerdo e átrio esquerdo. 


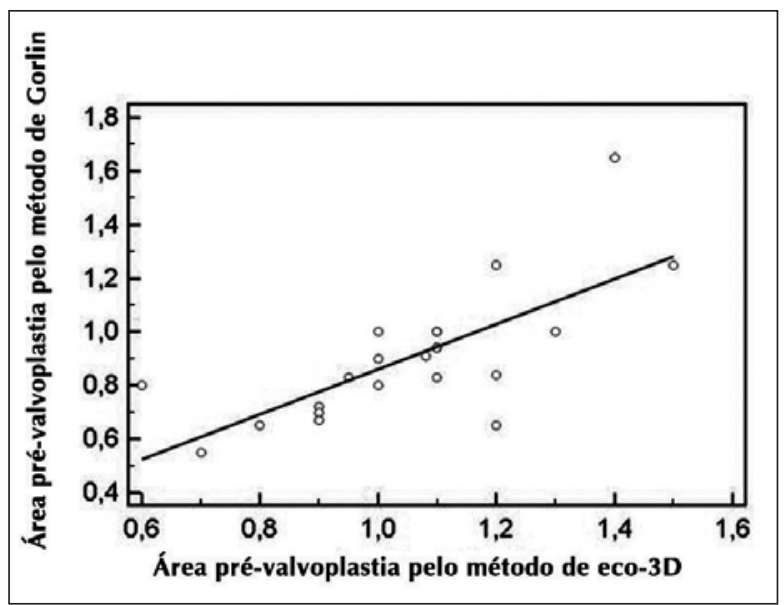

Figura 1 - Regressão linear demonstrando a correlação entre as áreas valvares mitrais obtidas pelo método invasivo e pelo método de ecocardiografia tridimensional pré-valvoplastia. eco-3D = ecocardiografia tridimensional.

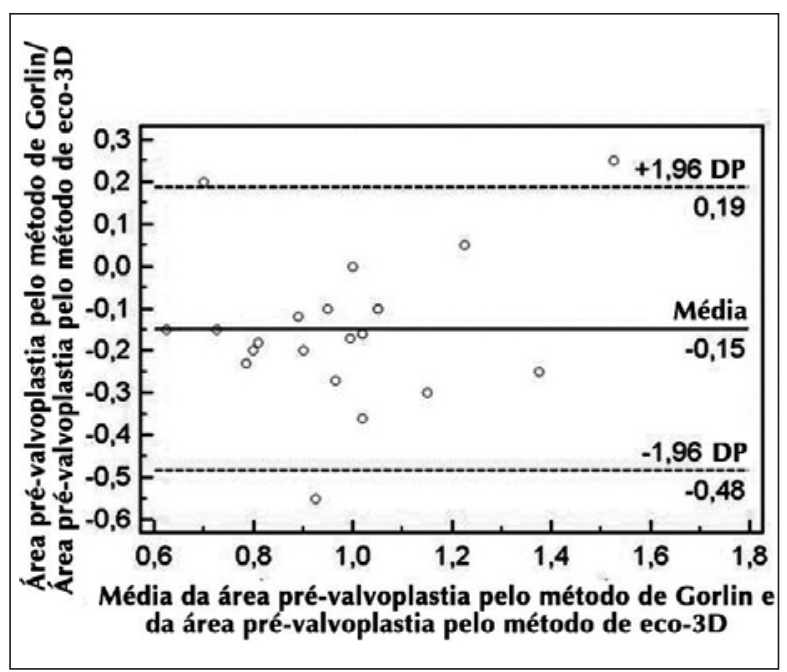

Figura 2 - Análise de concordância (teste de Bland \& Altman) entre o método invasivo e o método de ecocardiografia tridimensional pré-valvoplastia. $\mathrm{DP}=$ desvio padrão; eco-3D = ecocardiografia tridimensional.

estão em fibrilação atrial necessitam de planimetria e utiliza-se a média dos gradientes valvares em pelo menos 10 ciclos cardíacos. ${ }^{12}$ A fórmula de Gorlin é mais bem aplicada a pacientes em ritmo sinusal, sem insuficiência mitral, função ventricular esquerda normal, e nenhuma outra lesão valvar concomitante..$^{9-13}$

Neste estudo observou-se boa correlação da análise da área valvar mitral, tanto pré quanto pós-valvoplastia, aferida com a ecocardiografia tridimensional e com o método invasivo por meio da fórmula de Gorlin. A média da área valvar mitral obtida com o cálculo hemodinâmico foi de $1,73 \mathrm{~cm}^{2}$, enquanto a média obtida pela ecocardiografia tridimensional foi de $1,72 \mathrm{~cm}^{2}$. A

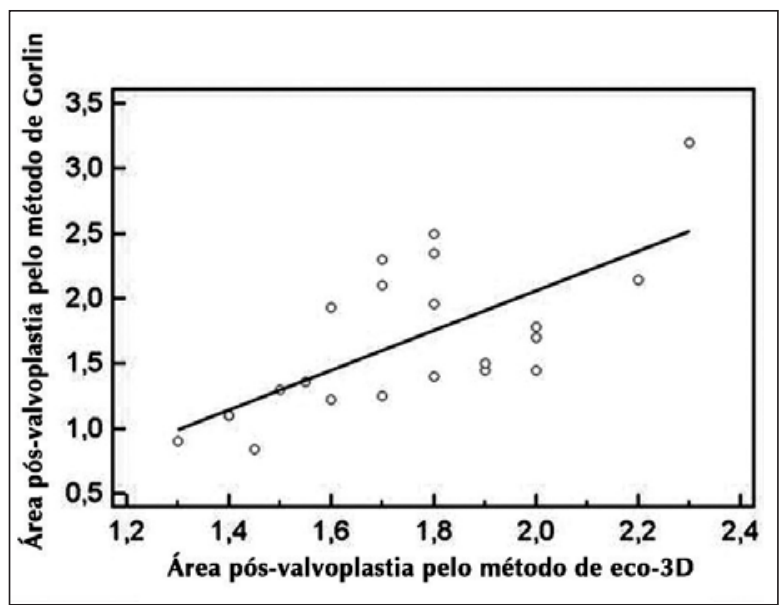

Figura 3 - Regressão linear demonstrando a correlação entre as áreas valvares mitrais obtidas pelo método invasivo e pelo método de ecocardiografia tridimensional pós-valvoplastia. eco-3D = ecocardiografia tridimensional.

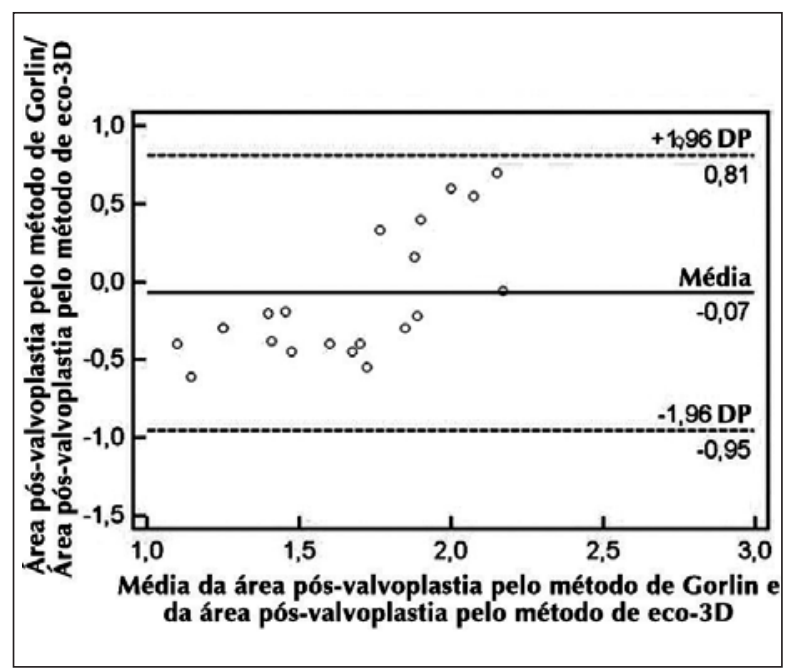

Figura 4 - Análise de concordância (teste de Bland \& Altman) entre o método invasivo e o método de ecocardiografia tridimensional pós-valvoplastia. DP = desvio padrão; eco-3D = ecocardiografia tridimensional.

correlação entre as áreas valvares mitrais obtidas por meio das medidas da fórmula de Gorlin e ecocardiografia tridimensional, pré e pós-valvoplastia por cateter-balão, foi de, respectivamente, $\mathrm{r}: 0,7638, \mathrm{P}<0,001$ e r: 0,6659, $\mathrm{P}<0,0019$. Nossos achados são concordantes com os de diversos estudos e os de uma meta-análise em que foi observada boa correlação ( $r$ : 0,83, variando entre $0,74$ e 0,89$)$ entre diversas medidas de análise morfofuncional da estenose mitral aferidas por meio do método invasivo e da ecocardiografia tridimensional. ${ }^{14-18}$

Os resultados deste estudo demonstram que foi observada boa concordância para a análise morfofuncional da valva mitral entre os dois métodos de análi- 
Dallan LAP, et al. Comparação das Medidas da Área Valvar Mitral Obtidas por Parâmetros Hemodinâmicos Invasivos e Ecocardiografia Tridimensional em Tempo Real Pré e Pós-Valvoplastia Mitral Percutânea. Rev Bras Cardiol Invasiva. 2010;18(3):321-6.

se, sendo a ecocardiografia tridimensional um método não-invasivo, com diferentes possibilidades de observação tanto temporal como espacial. É importante, no futuro, a escolha de modalidade de investigação cardíaca não-invasiva que alie custos baixos, grande reprodutibilidade, acurácia diagnóstica e disponibilidade de uso à população de baixa renda. ${ }^{17,18}$

\section{Limitações do estudo}

A fórmula de Gorlin é um método hemodinâmico que apresenta limitações, especialmente em situações em que ocorrem rápidas alterações hemodinâmicas, como o período pós-valvoplastia. A regurgitação mitral significativa e a presença de defeito septal atrial podem confundir as medições de fluxo transmitral. ${ }^{19-22}$

Já a ecocardiografia tridimensional é um método muito mais preciso e de mais fácil realização e avaliação dos resultados em indivíduos com função ventricular preservada e volumes dentro dos limites da normalidade. Há maior dificuldade da comparação em pacientes portadores de disfunção contrátil e dilatação ventricular, e também dentro de situações clínicas em que haja a presença de irregularidade do ritmo cardíaco.

Além disso, o número reduzido de pacientes incluídos no estudo faz com que a análise estatística possa apresentar imprecisões, sendo, portanto, necessários outros estudos com inclusão de maior número de pacientes.

Por último, é necessária a comparação da ecocardiografia tridimensional com outros métodos que avaliem a área valvar mitral, como planimetria e PHT.

\section{CONCLUSÃO}

A ecocardiografia tridimensional em tempo real é um exame adequado para avaliação e cálculo da área valvar mitral após procedimentos de valvoplastia mitral percutânea por cateter-balão, com valor fidedigno quando comparado ao consagrado cálculo da área valvar mitral obtido pelo método hemodinâmico invasivo pela fórmula de Gorlin.

\section{CONFLITO DE INTERESSES}

Os autores declararam inexistência de conflito de interesses relacionado a este manuscrito.

\section{REFERÊNCIAS}

1. Roelandt JRT, Yao J, Karsprazak JD. Three-dimensional echocardiography. Curr Opin Cardiol. 1998;13(6):386-98.

2. Kisslo J, Firek B, Ota T, Kang DH, Fleishman CE, Stetten G, et al. Real-time volumetric echocardiography: the technology and the possibilities. Echocardiography. 2000;17(8):773-9.

3. Ahmad M. Real-time three-dimensional echocardiography in assessment of heart disease. Echocardiography. 2001;18(1): 73-7.

4. Li J, Sanders SP. Three-dimensional echocardiography in congenital heart disease. Curr Opin Cardiol. 1999;14(1):53-9.

5. Vieira MLC, Pomerantzeff PMA, Leal SB, Mathias Junior W, Andrade JL, Ramires JAF. Ecocardiografia transesofágica tridi- mensional: acréscimo à informação diagnóstica e à análise anatômica. Rev Bras Ecocardiogr. 2003;1(1):47.

6. Vieira MLC, Ianni BM, Mady C, Encinas J, Pomerantzeff PM, Fernandes PP, et al. Mixoma de átrio esquerdo: avaliação ecocardiográfica tridimensional. Relato de caso. Arq Bras Cardiol. 2004;82(3):281-3.

7. De Simone R, Glombitza G, Vahl CF, Meinzer HP, Hagl S. Three-dimensional Doppler: techniques and clinical applications. Eur Heart J. 1999;20(8):619-27.

8. Vieira MLC, Nomura $\mathrm{CH}$, Tranchesi Junior B, Oliveira WA, Naccarato G, Serpa BS, et al. Fração de ejeção e volumes do ventrículo esquerdo medidos com eco 3D e com tomografia ultra-rápida. Arq Bras Cardiol. 2009;92(4):294-301.

9. Grossman W, Carabello BA. Calculation of stenotic valve orifice area. In: Baim DS. Grossman's cardiac catheterization, angiography, and intervention. $7^{\text {th }}$ ed. Philadelphia: Lippincott Williams and Wilkins; 2006.

10. Carabello BA. Advances in the hemodynamic assessment of stenotic cardiac valves. J Am Coll Cardiol. 1987;10(4):912-9.

11. Bonow RO, Carabello BA, Chatterjee $\mathrm{K}$, de Leon AC Jr, Faxon DP, Freed MD, et al. 2008 Focused update incorporated into the ACC/AHA 2006 guidelines for the management of patients with valvular heart disease: a report of the American College of Cardiology/American Heart Association Task Force on Practice Guidelines (Writing Committee to Revise the 1998 Guidelines for the Management of Patients With Valvular Heart Disease): endorsed by the Society of Cardiovascular Anesthesiologists, Society for Cardiovascular Angiography and Interventions, and Society of Thoracic Surgeons. Circulation. 2008;118(15):e523-661.

12. Kern MJ. The cardiac catheterization handbook. $4^{\text {th }}$ ed. St. Louis: Mosby; 2003.

13. Gorlin R, Gorlin SG. Hydraulic formula for calculation of the area of the stenotic mitral valve, other cardiac valves, and central circulatory shunts. Am Heart J. 1951;41(1):1-29.

14. Zamorano L, Isla JP, Sugeng L, Cordeiro P, Rodrigo JL, Almeria C, et al. Non-invasive assessment of mitral valve area during percutaneous balloon mitral valvuloplasty: role of real-time 3D echocardiography. Eur Heart J. 2004;25(23):2086-91.

15. Messika-Zeitoun D, Brochet E, Holmin C, Rosenbaum D, Cormier B, Serfaty JM, et al. Three-dimensional evaluation of the mitral valve area and commissural opening before and after percutaneous mitral commissurotomy in patients with mitral stenosis. Eur Heart J. 2007;28(1):72-9.

16. Zamorano J, Cordeiro P, Sugeng L, Perez de Isla L, Weinert L, Macaya C, et al. Real time 3D echocardiography for rheumatic mitral valve stenosis evaluation: An accurate and novel approach. J Am Coll Cardiol. 2004;43(11):2091-6.

17. de Agustin J, Nanda N, Gill EA, de Isla LP, Zamorano JL. The use of three-dimensional echocardiography for the evaluation of and treatment of mitral stenosis. Cardiol Clin. 2007;25(2):311-8.

18. Zamorano JL, de Agustin J. Three-dimensional echocardiography for assessment of mitral valve stenosis. Curr Opin Cardiol. 2009;24(5):415-9.

19. Nakatani S, Nagata S, Beppu S, Ishikura F, Tamai J, Yamagishi $M$, et al. Acute reduction of mitral valve area after percutaneous balloon mitral valvuloplasty: assessment with Doppler continuity equation method. Am Heart J. 1991;121(3 Pt 1):770-5.

20. Hammermeister KE, Murray JA, Blackmon JR. Revision of Gorlin constant for calculation of mitral valve area from left heart pressures. Br Heart J. 1973;35(4):392-6.

21. Flachskampf FA, Weyman AE, Guerrero JL, Thomas JD. Influence of orifice geometry and flow rate on effective valve area: an in vitro study. J Am Coll Cardiol. 1990;15(5):1173-80.

22. Klarich KW, Rihal CS, Nishimura RA. Variability between methods of calculating mitral valve area: simultaneous Doppler echocardiographic and cardiac catheterization studies conducted before and after percutaneous mitral valvuloplasty. J Am Soc Echocardiogr. 1996;9(5):684-90. 